\title{
Side population cells from long-term passage non-small cell lung cancer cells display loss of cancer stem cell-like properties and chemoradioresistance
}

\author{
HAO GU ${ }^{1 *}$, XIN-YU WU ${ }^{2 *}$, RUI-TAI FAN ${ }^{1}$, XIN WANG $^{1}$, YOU-ZHONG GUO ${ }^{1}$ and RUI WANG ${ }^{1}$ \\ ${ }^{1}$ Department of Radiation Oncology, The First Affiliated Hospital of Zhengzhou University, Zhengzhou, Henan 450052; \\ ${ }^{2}$ Department of Nuclear Medicine, Henan Provincial People's Hospital and \\ The People's Hospital of Zhengzhou University, Zhengzhou, Henan 450003, P.R. China
}

Received April 7, 2015; Accepted May 13, 2016

DOI: $10.3892 / \mathrm{ol} .2016 .4934$

\begin{abstract}
The side population (SP) assay is a widely used method for isolating stem cell-like cells from cancer cell lines and primary cells. The cancer cells used in different laboratories have been passaged for different generations. Emerging evidence revealed that repeated passaging of cell lines for multiple generations frequently leads to change of characteristics. Thus, it is worth investigating the effects of repeated passaging on the biological and functional properties of the enriched SP fraction from early- and late-passage cells. The present study reports that the cancer stem cell (CSC) characteristics, including increased frequency of tumor-initiating and self-renewal capacity, and resistance to the chemotherapy agent doxorubicin and ionizing radiation, was diminished in SP cells from late-passage non-small cell lung cancer (NSCLC) cells. This finding revealed that the SP from long-term passage NSCLC cells was not consistently enriched for stem cell-like cancer cells, and low-passage cell lines and primary cancer cells are therefore recommended in the CSCs field.
\end{abstract}

\section{Introduction}

Lung cancer is the most common cause of cancer-associated mortality, and its incidence has been steadily rising worldwide over the past 20 years (1-3). Lung cancer is divided into small cell lung cancer (SCLC) and non-SCLC (NSCLC) (3). The most common forms of NSCLC include adenocarcinoma, squamous

Correspondence to: Professor Rui-Tai Fan, Department of Radiation Oncology, The First Affiliated Hospital of Zhengzhou University, 1 Jianshe East Road, Zhengzhou, Henan 450052, P.R. China

E-mail: fanruitai@hotmail.com

*Contributed equally

Key words: side population, cancer stem cell, early passage, late passage, tumorigenicity, resistance cell carcinoma and large cell carcinoma, which comprises $\sim 80 \%$ of all lung cancer cases $(1,2)$. Patients with lung cancer frequently present with metastases, regardless of the primary tumor size at the initial diagnosis, and always have a high rate of relapse following treatment (3). Therapeutic approaches for lung cancer are multifactorial and include surgery, immunotherapy, radiotherapy and targeted therapy (4). Despite the widespread use of multimodal treatment, the overall five-year survival rate for such tumors is $<15 \%$ (2).

Previous studies revealed that numerous malignant tumors such as lung cancer, are composed of diverse cell types with distinct proliferative and differential capacities (5-7). Thus, the emerging reasonable explanation is the existence of a rare subpopulation of cells that appear to be cancer stem cells (CSCs), and which may contribute in certain cases to resistance to cancer therapy and relapse (6-8). In fact, several studies have suggested that a stem-like subpopulation derived from lung cancer cell lines and tumor specimens was isolated by flow cytometry, according to the detection of side population (SP) phenotypes (5).

Ho et al (5) reported that NSCLC cell lines, including H460, H23, HTB-58, A549, H441 and H2170, contained SP cells ranging from 1.5 to $6.1 \%$ of the total viable cell population. In another study by Salcido et al (9), SCLC cell lines (H146 and H526) were observed to comprise $0.7-1.3 \%$ of SP cells, while the NSCLC cell lines A549 and H460 contained 2.59 and $4.00 \%$ of SP cells, respectively. Sung et al (10) reported that $24.44 \%$ of A549 cells were classified as SP cells. Notably, the NSCLC cell line A549 used in the aforementioned studies exhibited a significantly different SP fraction, ranging from 2.59 to $24.44 \%(5,9,10)$. Those results indicate that the frequency of the SP fraction appears to be highly variable between different lung cancer cell lines and among the same type of cells, which may be associated with the use of lung cancer sublines passaged for different generations in individual laboratories. Emerging evidence revealed that repeated passaging of cell lines for multiple generations frequently leads to change of characteristics, including alterations in cell morphology, growth rates, protein expression and cell signaling, and acquisition of genetic aberrations (11-13). Generally, established cancer cell lines have usually been 
passaged many times in vitro within one laboratory (14). Based on these findings, it is worth investigating the effects of repeated passaging on the biological and functional properties of the enriched SP fraction from early- and late-passage cells.

In order to test this hypothesis, A549 and NSCLC SP cells from low- and long-term passage cells were isolated by flow cytometry based on ATP-binding cassette (ABC) sub-family G member 2 efflux pump-mediated Hoechst 33342 dye exclusion. The isolated SP cells were used to investigate whether increasing cell passage could alter their CSC-associated biological and functional properties. This may aid to explain previous unclear results and to better understand the biology of NSCLC CSCs.

\section{Materials and methods}

Cell line and clinical sample. The human NSCLC cell line A549 was obtained from the American Type Culture Collection (Manassas, VA, USA) and maintained in complete medium consisting of RPMI-1640 supplemented with $10 \%$ (v/v) fetal bovine serum (FBS; HyClone; GE Healthcare Life Sciences, Chalfont, UK) and $1 \%$ penicillin-streptomycin (Invitrogen; Thermo Fisher Scientific, Inc., Waltham, MA, USA) in a humidified $37^{\circ} \mathrm{C}$ incubator with $5 \% \mathrm{CO}_{2}$.

Tumor specimens were obtained from the consenting patient according to the Internal Review and Ethics Board of The First Affiliated Hospital of Zhengzhou University (Zhengzhou, China). Tumor was obtained at radical surgery for a 52-year-old male NSCLC patient. The fresh tumor was minced, suspended in Dulbecco's modified Eagle medium (DMEM)/F12 medium (Invitrogen; Thermo Fisher Scientific, Inc.) and mixed with $300 \mathrm{U} / \mathrm{ml}$ collagenase I (Invitrogen; Thermo Fisher Scientific, Inc.) and $300 \mathrm{U} / \mathrm{ml}$ hyaluronidase (Calbiochem; EMD Millipore, Billerica, MA, USA), followed by overnight incubation at $37^{\circ} \mathrm{C}$ with $5 \% \mathrm{CO}_{2}$. Enzymatically disaggregated suspensions were filtered with a $40-\mu \mathrm{m}$ cell strainer and washed twice with phosphate-buffered saline (PBS), and red blood cells were then removed using Ammonium Chloride Lysing Solution (Sigma-Aldrich, St. Louis, MO, USA). The resulting single tumor cells were cultured in DMEM/F12 supplemented with $10 \% \mathrm{FBS}$ at $37^{\circ} \mathrm{C}$ in a humidified atmosphere containing $5 \% \mathrm{CO}_{2}$.

The A549 cell line and the fresh isolated NSCLC cells were passaged for 50 generations (1 passage every 4 days). The cells at the 2 nd (low passage) and 50th (long-term passage) passages were analyzed.

Analysis and isolation of SP cell fraction. SP analysis was performed as described by Goodell et al (15) with slight modifications. Briefly, A549 and NSCLC cells at the 2nd and 50th passages were digested with $0.25 \%$ trypsin (Sigma-Aldrich), washed twice with PBS and resuspended in pre-warmed RPMI-1640 culture medium (Macgene Biotechnology Ltd., Beijing, China) supplemented with $2 \%$ FBS and $2 \mathrm{mM}$ 4-(2-hydroxyethyl)-1-piperazineethanesulfonic acid at a density of $1 \times 10^{6}$ cells $/ \mathrm{ml}$. Then, the cells were incubated for an additional $90 \mathrm{~min}$ at $37^{\circ} \mathrm{C}$ in a shaking bath with $5 \mu \mathrm{g} / \mathrm{ml}$ Hoechst 33342 dye (Invitrogen; Thermo Fisher Scientific, Inc.). Control cells were incubated with $50 \mathrm{mM}$ verapamil (Sigma-Aldrich) for $15 \mathrm{~min}$ at $37^{\circ} \mathrm{C}$ prior to the addition of Hoechst dye. Upon staining, the cells were washed twice with ice-cold PBS and resuspended in cold PBS. Flow cytometry analysis and cell sorting were conducted on a FACSAria II flow cytometer (BD Biosciences, Franklin Lakes, NJ, USA). Hoechst 33342 was excited with ultraviolet (UV) light at $355 \mathrm{~nm}$, and fluorescence emission was detected with 450/BP50 (Hoechst blue) and a 660/BP50 (Hoechst red) optical filters (BD Biosciences). The collected SP cells were used to perform all subsequent experiments.

Clone formation assay. A total of 250 sorted SP cells were plated on a 6-well plate (Corning Incorporated, Corning, NY, USA) in triplicate and cultured with DMEM/F12 supplemented with $10 \%$ FBS for 10 days. The cells were then fixed and stained with $0.5 \%$ crystal violet. Colonies containing $>50$ cells were counted by eye.

Sphere formation assay. Sorted SP cells were plated at $5 \times 10^{3}$ cells/well in serum-free DMEM/F12 supplemented with $20 \mathrm{ng} / \mathrm{ml}$ epidermal growth factor (Invitrogen; Thermo Fisher Scientific, Inc.,), $10 \mathrm{ng} / \mathrm{ml}$ human recombinant basic fibroblast growth factor (Invitrogen; Thermo Fisher Scientific, Inc.) and B-27 ${ }^{\circledR}$ Supplement (Invitrogen; Thermo Fisher Scientific, Inc.). The medium was changed every other day. After 2 weeks in culture, colonies containing $>20$ cells were counted using a microscope (Eclipse TE2000-S; Nikon Corporation, Tokyo, Japan).

Reverse transcription-quantitative polymerase chain reaction $(R T-q P C R)$ analysis. Total RNA was obtained from freshly sorted SP cells using RNAiso Plus (Takara Biotechnology Co., Ltd., Dalian, China) and RT reactions were performed using PrimeScript ${ }^{\mathrm{TM}}$ RT Master Mix (Perfect Real Time) kit according to the manufacturer's protocol (Takara Biotechnology Co., Ltd.). RT was performed under the following conditions: $30^{\circ} \mathrm{C}$ for $10 \mathrm{~min}, 95^{\circ} \mathrm{C}$ for $1 \mathrm{~h}$ and $4^{\circ} \mathrm{C}$ for 5 min. qPCR was performed using SYBR ${ }^{\circledR}$ Green I Master Mix kit (Takara Biotechnology Co., Ltd.) on an iQ5 real-time PCR detection system (Bio-Rad Laboratories, Inc., Hercules, CA, USA) under the following conditions: $95^{\circ} \mathrm{C}$ for 2 min followed by 45 cycles of denaturation at $95^{\circ} \mathrm{C}$ for $15 \mathrm{sec}$, annealing at $58^{\circ} \mathrm{C}$ for $20 \mathrm{sec}$ and extension at $72^{\circ} \mathrm{C}$ for $30 \mathrm{sec}$. The relative amounts of messenger RNA (mRNA) were calculated from the values of the comparative threshold cycle (16) using glyceraldehyde 3-phosphate dehydrogenase as a control. The sequences of the primers used are indicated in Table I.

Tumorigenicity assay and in vivo micro-positron emission tomography (PET) imaging. Animal experiments were performed in accordance to the Guide for the Care and Use of Laboratory Animals of The First Affiliated Hospital of Zhengzhou University. Briefly, sorted SP cells suspended in $100 \mu 1$ Matrigel (BD Biosciences) were injected subcutaneously into 5-week-old female non-obese diabetic/severe combined immunodeficiency mice ( $n=48$; Institute of Laboratory Animal Science, Zhengzhou University). The number of injected cells is indicated in Table II. Tumor growth was monitored 4 weeks after transplantation using a MOSAIC small animal PET scanner (Philips Medical Systems, Inc., Bothell, WA, USA). For microPET imaging, the mice had been fasting for $10 \mathrm{~h}$ prior to ${ }^{18} \mathrm{~F}$-fluorodeoxyglucose (FDG) injections but allowed 
Table I. Sequences of the primers used in reverse transcription-quantitative polymerase chain reaction.

\begin{tabular}{|c|c|c|}
\hline Gene (transcript number) & Sequence $\left(5^{\prime}-3^{\prime}\right)$ & Product siz \\
\hline ABCG2 & F: CACGTGATTCTTCCACAAGCC & 74 \\
\hline (NM_004827) & R: CATGTACTGGCGAAGAATATTTGGT & \\
\hline Bmi1 & F: AAATGCTGGAGAACTGGAAAG & 124 \\
\hline (NM_005180) & R: CTGTGGATGAGGAGACTGC & \\
\hline E-cadherin & F: ATTCTGATTCTGCTGCTCTTG & 400 \\
\hline (NM_004360) & R: AGTAGTCATAGTCCTGGTCTT & \\
\hline GAPDH & F: AATTGAGCCCGCAGCCTCCC & 153 \\
\hline (NM_002046) & R: CCAGGCGCCCAATACGACCA & \\
\hline Nanog & F: ATTCAGGACAGCCCTGATTCTTC & 76 \\
\hline (NM_024865) & R: TTTTTGCGACACTCTTCTCTGC & \\
\hline N-cadherin & F: CTCCTATGAGTGGAACAGGAACG & 121 \\
\hline (NM_001792) & R: TTGGATCAATGTCATAATCAAGTGCTGTA & \\
\hline Oct4 & F: GTGGAGAGCAACTCCGATG & 86 \\
\hline (NM_002701) & R: TGCTCCAGCTTCTCCTTCTC & \\
\hline Snail & F: GAGGCGGTGGCAGACTAG & 159 \\
\hline (NM_005985) & R: GACACATCGGTCAGACCAG & \\
\hline Sox2 & F: CGAGTGGAAACTTTTGTCGGA & 74 \\
\hline (NM_003106) & R: TGTGCAGCGCTCGCAG & \\
\hline Twist & F: CGGGAGTCCGCAGTCTTA & 130 \\
\hline (NM_000474) & R: TGAATCTTGCTCAGCTTGTC & \\
\hline
\end{tabular}

F, forward; R, reverse; ABCG2, ATP-binding cassette sub-family G member 2; Bmi1, B lymphoma Mo-MLV insertion region 1 homolog; GAPDH, glyceraldehyde 3-phosphate dehydrogenase; Oct4, octamer-binding transcription factor 4; Sox 2, sex determining region Y-box 2.

Table II. Tumorigenicity of 2nd and 50th passage cells in A549 and NSCLC cells.

\begin{tabular}{lcccc}
\hline & \multicolumn{4}{c}{ Injected cell number } \\
\cline { 2 - 5 } Cell & $1 \times 10^{2}$ & $1 \times 10^{3}$ & $1 \times 10^{4}$ & $1 \times 10^{5}$ \\
\hline A549 & & & & \\
2nd & $0 / 3$ & $0 / 3$ & $2 / 3$ & $3 / 3$ \\
50th & $0 / 3$ & $0 / 3$ & $0 / 3$ & $2 / 3$ \\
NSCLC & & & & \\
2nd & $1 / 3$ & $1 / 3$ & $3 / 3$ & $3 / 3$ \\
50th & $0 / 3$ & $0 / 3$ & $1 / 3$ & $3 / 3$ \\
\hline
\end{tabular}

NSCLC, non-small cell lung cancer.

free access to water. Upon being intraperitoneally anesthetized with $100 \mathrm{mg} / \mathrm{kg}$ pentobarbital (Sigma-Aldrich), each mouse was injected intravenously with $\sim 3.7 \mathrm{MBq}{ }^{18} \mathrm{~F}-\mathrm{FDG}$ (which was provided by the Department of Nuclear Medicine of The First Affiliated Hospital of Zhengzhou University). Then, the mice were euthanized to confirm tumor formation.

Radiation sensitivity assay. Briefly, 500-10,000 SP cells were plated on $25 \mathrm{~cm}^{2}$ flasks in triplicate for each experiment.
Table III. Radiobiological parameters of 2nd and 50th passage cells in A549 and NSCLC cells.

\begin{tabular}{ccccc}
\hline Cell & $\mathrm{N}^{\mathrm{a}}$ & $\mathrm{D}_{0}(\mathrm{~Gy})^{\mathrm{a}}$ & $\mathrm{D}_{\mathrm{q}}(\mathrm{Gy})^{\mathrm{a}}$ & SER D $_{\mathrm{q}}$ \\
\hline A549 & & & & 1.120 \\
2nd & $1.291 \pm 0.027$ & $2.639 \pm 0.127$ & $0.674 \pm 0.062$ & \\
50th & $1.303 \pm 0.035$ & $2.273 \pm 0.141$ & $0.602 \pm 0.089$ & \\
NSCLC & & & & 1.335 \\
2nd & $1.723 \pm 0.023$ & $2.933 \pm 0.059$ & $1.596 \pm 0.026$ & \\
50th & $1.754 \pm 0.061$ & $2.128 \pm 0.072$ & $1.196 \pm 0.064$ & \\
\hline
\end{tabular}

${ }^{\text {a }}$ Values are the mean \pm standard error of the mean $(n=3)$. N, extrapolation number (parameter to measure the width of the shoulder of the survival curve); $\mathrm{D}_{0}$, mean lethal dose; $\mathrm{D}_{\mathrm{q}}$, quasi-threshold dose; SER, sensitization enhancement ratio; NSCLC, non-small cell lung cancer.

Then, the cells were exposed to a ${ }^{60} \mathrm{Co}$ laboratory irradiator (GammaCell 220; MDS Nordion, Inc., Ontario, Canada) for the time required to generate a dose curve of $0,2,4,6$ and 8 Gy. The control was sham irradiated SP cells. Following irradiation, the cells were incubated for an additional 10 days, prior to be fixed with $100 \%$ carbinol and stained with $0.5 \%$ crystal violet. The surviving fraction (SF) was calculated as follows: Plating efficiency $(\mathrm{PE})=($ colony number/inoculated 
A

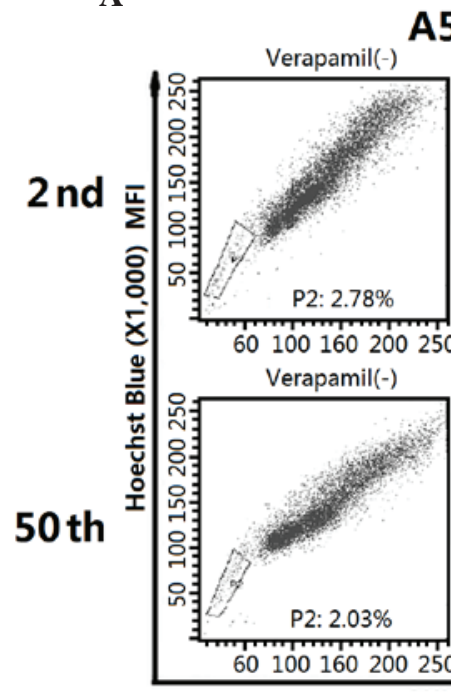

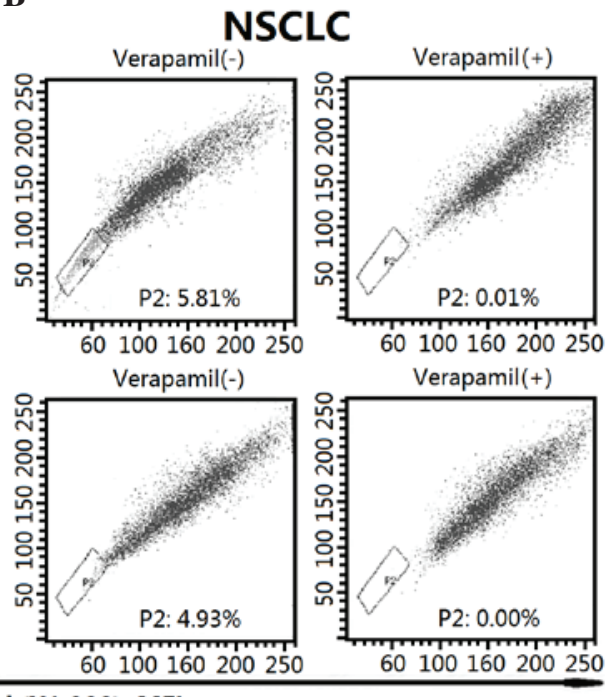

Figure 1. Isolation of the SP fraction from different passage A549 and NSCLC cells. The SPs from the 2nd and 50th passage of (A) A549 and (B) NSCLC cells were isolated by flow cytometry. SP, side population; NSCLC, non-small cell lung cancer.

cell number) $\mathrm{x} 100 \% . \mathrm{SF}=\mathrm{PE}_{\text {tested group }} / \mathrm{PE}_{0 \text { Gy group }} \times 100 \%$. The cell survival curves were fitted using the following single-hit multi-target formula: $\mathrm{SF}=1-(1-\mathrm{e}-\mathrm{D} / \mathrm{D} 0)^{\mathrm{N}}(17)$. The radiobiological parameters of cellular radiosensitivity (mean lethal dose, $\mathrm{D}_{0}$ ), the capacity for sublethal damage repair (quasi-threshold dose, $\mathrm{D}_{\mathrm{q}}$ ) and the extrapolation number $(\mathrm{N})$ were calculated and then used to calculate the SF after irradiation at a dose of $2 \mathrm{~Gy}\left(\mathrm{SF}_{2}\right)$ and the sensitization enhancement ratio (SER) according to the following formula: $S E R=D_{q} 2 n d / D_{q} 50$ th (Table III).

Drug sensitivity assay. Sulforhodamine B (SRB) assay was used to determine the sensitivity of SP cells to doxorubicin. Cells were plated at a density of $1 \times 10^{4}$ cells/well in a 96-well plate. After incubation for $24 \mathrm{~h}$, the cells were treated with 250, 500 and 1,000 $\mathrm{nM}$ doxorubicin (Sigma-Aldrich). Then, the cells were fixed with $10 \%(\mathrm{w} / \mathrm{v})$ trichloroacetic acid at $4^{\circ} \mathrm{C}$ for $1 \mathrm{~h}$ and stained for 30 min with $0.4 \%(\mathrm{w} / \mathrm{v})$ SRB (Sigma-Aldrich) dissolved in $1 \%$ acetic acid. The protein-bound dye was subsequently dissolved in $10 \mathrm{mmol} / \mathrm{l}$ Tris. The optical density of the cells was determined at a wavelength of $540 \mathrm{~nm}$ in an iMark ${ }^{\mathrm{TM}}$ microplate absorbance reader (Bio-Rad Laboratories, Inc.).

Apoptosis assay. For quantitative analysis of apoptosis, the sorted SP cells were pretreated with $500 \mathrm{nM}$ doxorubicin for $12 \mathrm{~h}$ or exposed to $2 \mathrm{~Gy}$ irradiation. Then, $1 \times 10^{6}$ cells were immediately suspended in $200 \mu 1$ annexin V-fluorescein isothiocyanate (FITC) binding buffer (Annexin V FITC Apoptosis Detection kit; BD Biosciences) containing $10 \mu 1$ annexin V-FITC. After 15 min of incubation in the dark at room temperature, the cells were diluted with $300 \mu \mathrm{l}$ annexin V-FITC binding buffer containing $5 \mu \mathrm{l}$ propidium iodide (PI) and immediately analyzed using a FACSCalibur ${ }^{\mathrm{TM}}$ flow cytometer (BD Biosciences). The annexin V-FITC and PI emissions were detected using 515 and $560 \mathrm{~nm}$ filters, respectively, following excitation with a 488 -nm UV light. The number of apoptotic cells were counted using CellQuest 3.1 software (BD Biosciences).

Statistical analysis. Data were expressed as means \pm standard error of the mean $(n=3-6)$. The difference between SP cells were analyzed using the Student's $t$ test. Statistical analyses were performed using SPSS software version 17 (SPSS, Inc., Chicago, IL, USA). P $<0.05$ was considered to indicate a statistically significant difference.

\section{Results}

Isolation of SP fraction from different passage A549 and NSCLC cells. In order to investigate the functional properties of the SP fraction from low and long-term passage cells, the SP subpopulation was first isolated by flow cytometry. As indicated in Fig. 1A, A549 cell lines contained a similar fraction of SP cells in the 2nd (2.78\%) and 50th $(2.03 \%)$ passage cells. For early- and late-passage NSCLC cells, SP cells accounted for 5.81 and $4.93 \%$ of the whole population, respectively (Fig. 1B). The SP fraction decreased significantly in the presence of verapamil.

The stem cell-like properties of the SP fraction from A549 and NSCLC cells were diminished upon long-term passage. To determine the differences in the clonogenic capacity of the SP fraction from 2nd and 50th passage cells, a clone formation assay was performed. The results indicated that the mean clone formation efficiency was $79.81 \pm 5.71$ and $66.84 \pm 3.47 \%$ in early and late passage A549 cells, respectively (Fig. 2A and B). For NSCLC cells, the percentage of formed clones in 2nd and 50th passage cells was $78.27 \pm 2.26$ and $61.45 \pm 3.72 \%$, respectively (Fig. 2A and B).

Sphere formation has been recognized as a defined characteristic of CSCs that reflects the potential of self-renewal ability (18). Thus, the sphere formation capacity was evaluated. As shown in Fig. 2C and D, the sphere formation efficiency of 2 nd and 50th passage sublines was $15.70 \pm 2.42$ and 

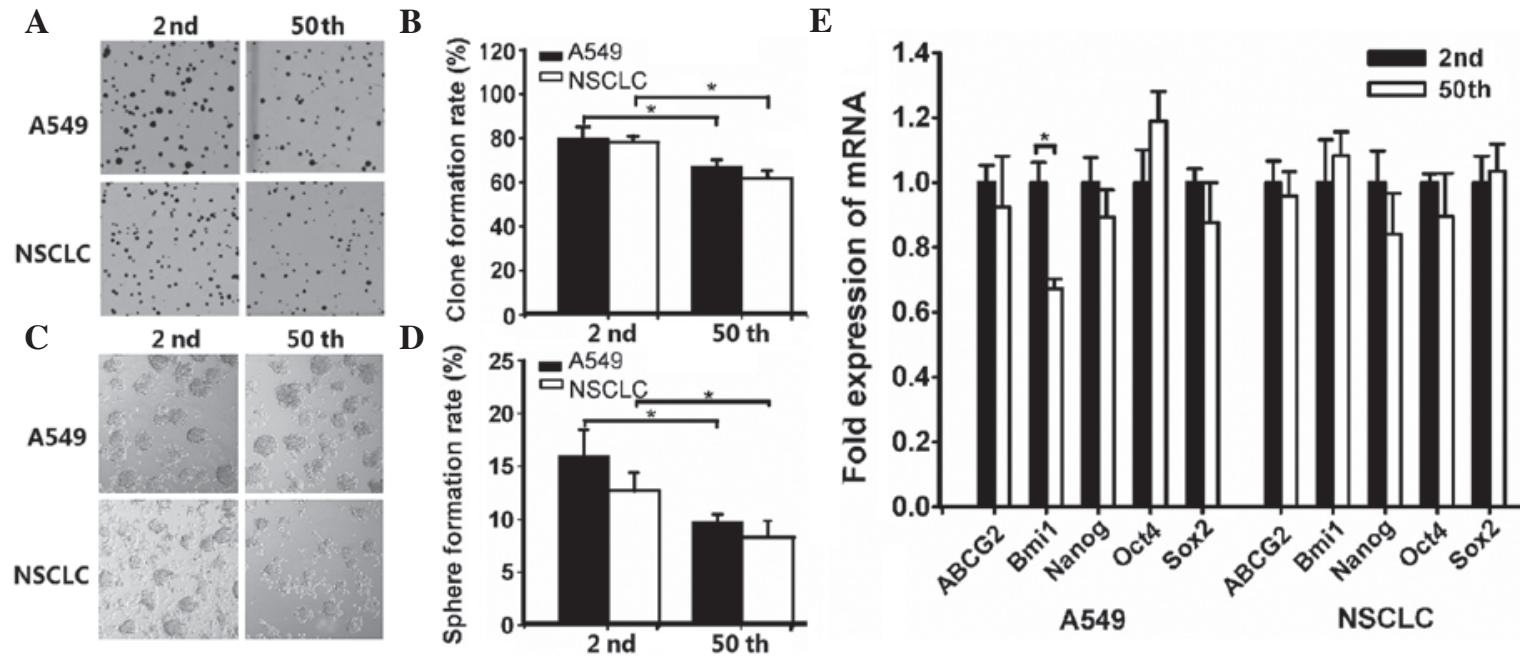

Figure 2. The stem cell-like properties of the side population fraction from A549 and non-small cell lung cancer cells were diminished following long-term passage. Clone formation was (A) photographed (magnification, x1) and (B) calculated after 10 days culture. The formed spheres on day 14 were (C) photographed (magnification, $\mathrm{x} 4$ ) and (D) counted to calculate the rate of formation. (E) Reverse transcription-quantitative polymerase chain reaction analysis of stemness genes expression ("P<0.05). NSCLC, non-small cell lung cancer; mRNA, messenger RNA; ABCG2, ATP-binding cassette sub-family G member 2; Bmi1, B lymphoma Mo-MLV insertion region 1 homolog; Oct4, octamer-binding transcription factor 4; Sox 2, sex determining region Y-box 2.
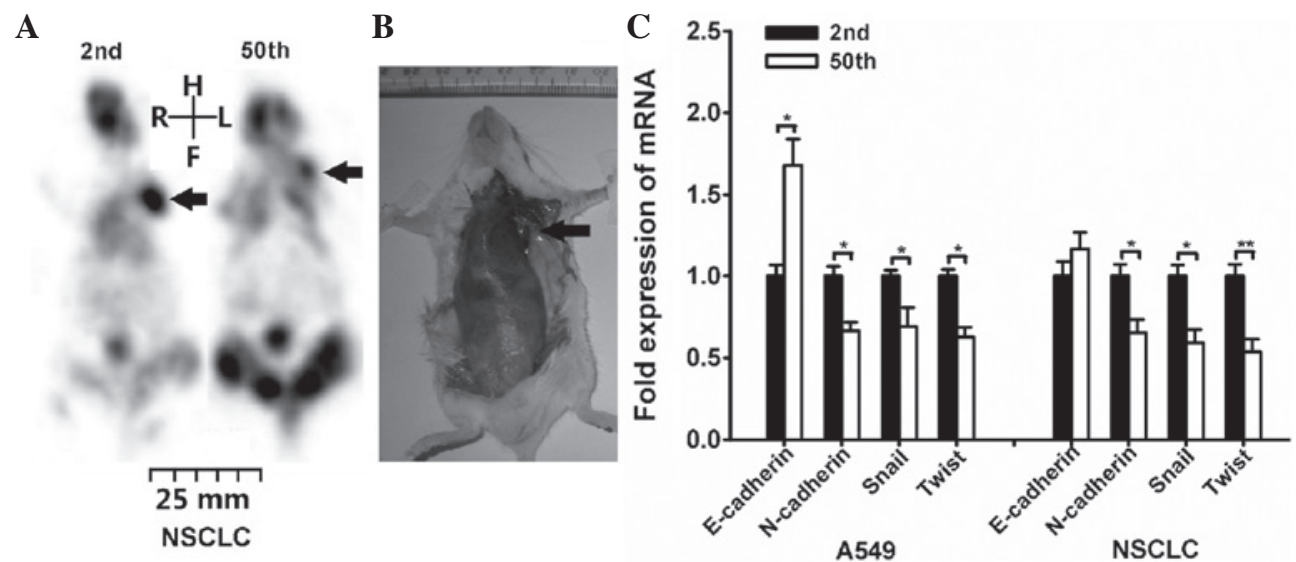

Figure 3. SP fraction exhibited decreased tumorigenicity and invasion capacity after long-term passage. NSCLC SP cells were injected subcutaneously on the left side flank region of NOD/SCID mice. (A) $1 \times 10^{4}$ NSCLC SP cells were injected into NOD/SCID mice, and the tumor growth was monitored on day 28 by micro-positron emission tomography imaging (as indicated by arrow). Then, the mice were euthanized to confirm tumor formation. (B) The arrow indicates the tumor-associated lesion induced by $1 \times 10^{5}$ 2nd passage NSCLC SP cells injection. (C) Reverse transcription-quantitative polymerase chain reaction analysis of the expression of epithelial-mesenchymal transition-associated genes ( $\left.{ }^{*} \mathrm{P}<0.05,{ }^{* * *} \mathrm{P}<0.01\right)$. NOD, non-obese diabetic; SCID, severe combined immunodeficiency; NSCLC, non-small cell lung cancer; mRNA, messenger RNA; SP, side population; R, right; L, left; H, head; F, foot.

$9.45 \pm 0.68 \%$ in A549 cells, and $12.46 \pm 1.63$ and $8.08 \pm 1.50 \%$ in NSCLC cells, respectively.

Based on the notion that CSCs frequently have conserved stem and progenitor cell phenotypes (19), the expression of embryonic stem (ES) cell-associated genes was evaluated. As shown in Fig. 2E, RT-qPCR analysis revealed that the mRNA expression of 2nd passage SP cells (with the exception of $\mathrm{B}$ cell-specific Moloney murine leukemia virus integration site 1 expression in A549 cells) had no significant differences compared with 50th passage SP cells.

The present study revealed that SP fraction exhibits decreased tumorigenicity and invasion capacity following long-term passage. Serial transplantation is the most widely accepted assay and recommended by the American Association for Cancer Research to determine stem cell properties $(20,21)$. Thus, the tumorigenic potential of the SP subpopulation within A549 and NSCLC cells was investigated. As depicted in Table II, the results indicated that the tumor-initiating frequency was decreased after long-term passage. This result was also confirmed by microPET imaging (Fig. 3A). The SP cells were injected subcutaneously on the left side flank region, and a formed tumor could be clearly identified (as indicated by the arrow in the image). In order to confirmed tumor formation on microPET scanning, the mice were euthanized after microPET imaging. The tumor-associated lesion (indicated by the arrow) was observed in the left region of certain mice following $1 \times 10^{5} 2$ nd NSCLC SP cells injection (Fig. 3B).

Additionally, the expression of genes associated with tumor cell migration was evaluated. The migration and invasion abilities of CSCs have been frequently associated with the epithelial-mesenchymal transition (EMT) $(22,23)$. 

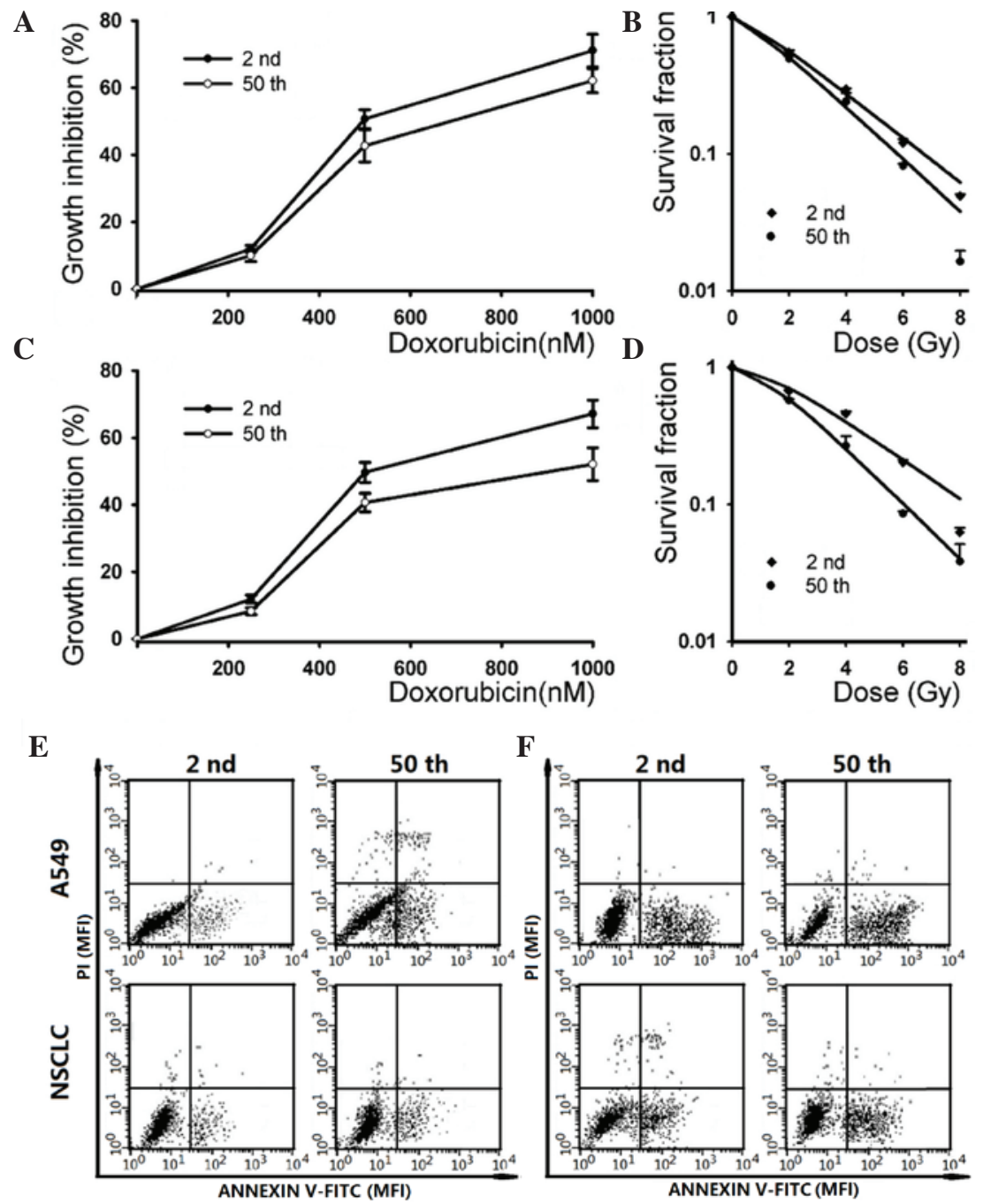

Figure 4. The SP fraction derived from different passage cells displayed distinct chemo- and radio-sensitivity. (A) SP cells from A549 cells were treated with different concentrations of doxorubicin $(0,250,500$ and $1,000 \mathrm{nM})$, and cytotoxicity was determined by SRB assay ("P<0.05). (B) SP cells within A549 cells were irradiated with a dose of $0,2,4,6$ and 8 Gy. The survival curves represent the data as fitted by the single-hit multi-targets model ("P<0.05). (C) SP cells from NSCLC cells were treated with different concentrations of doxorubicin $(0,250,500$ and 1,000 nM), and cytotoxicity was determined by SRB assay $($ ( $\mathrm{P}<0.05)$. (D) SP cells within NSCLC cells were irradiated with a dose of $0,2,4,6$ and 8 Gy. The survival curves represent the data as fitted by the single-hit multi-targets model ( $\left.{ }^{*} \mathrm{P}<0.05,{ }^{* *} \mathrm{P}<0.01\right)$. SP cells were (E) exposed to $2 \mathrm{~Gy}$ irradiation or (F) pretreated with $500 \mathrm{nM}$ doxorubicin for $12 \mathrm{~h}$, and the apoptosis rate was determined by annexin V-FITC/propidium iodide staining. NSCLC, non-small cell lung cancer; PI, propidium iodide; SP, side population; SRB, sulforhodamine B; FITC, fluorescein isothiocyanate; MFI, mean fluorescence intensity.

Thus, the expression of EMT-associated genes, including E-cadherin, N-cadherin, Snail and Twist, was investigated. RT-qPCR analysis indicated that N-cadherin, Snail and Twist genes were expressed higher in 2nd passage cells than in 50th passage cells (Fig. 3C). For E-cadherin, its mRNA expression was increased in 50th passage A549 cells, while displayed no significant differences in NSCLC cells.

The SP fraction derived from different passage cells exhibits distinct chemo- and radio-sensitivity $(5,9,10)$. Increasing evidence supports the notion that CSCs play a crucial role in therapeutic resistance, which results in tumor relapse $(24,25)$. To determine whether long-term passage affects the sensitivity of the SP subpopulation to chemo- and radiotherapy, the cytotoxicity of doxorubicin and ionizing radiation on different passage A549 and NSCLC SP cells were evaluated. The SRB assay indicated that the 2 nd passage subline was more resistent to high doses of doxorubicin $(1,000 \mathrm{nM})$ than the 50th passage subline in A549 cells (Fig. 4A). For NSCLC cells, the 50th passage subline exhibited more sensitivity to high doses of doxorubicin $(1,000 \mathrm{nM})$ than the 2 nd passage subline, while it had no significant difference when low doses of doxorubicin were used. Additionally, the A549 cells exhibited no significant difference in their sensitivity to low doses of doxorubicin.

The radiation-induced dose-dependent survival curve of 2nd and 50th passage SP cells are also presented in Fig. 4. The results indicated that the 2 nd passage cells exhibited more resistance than the 50th passage cells in A549 (exposure to 8 Gy irradiation) and NSCLC (exposure to 6 and 8 Gy irradiation) SP cells. The SF after 2 Gy irradiation was $55.84 \pm 1.91 \%$ for 2 nd passage cells vs. $50.26 \pm 2.24 \%$ for 50 th passage cells in the A549 cell line, and $70.32 \pm 1.52 \%$ for 2 nd passage cells vs. $58.13 \pm 1.21 \%$ for 50th passage cells in NSCLC cells. By application of the single-hit multi-target model, the values of $\mathrm{N}, \mathrm{D}_{0}$ and $\mathrm{D}_{\mathrm{q}}$ of $2 \mathrm{nd}$ and 50th passage SP cells were analyzed (Table III). Those radiobiological parameters and the $S E R D_{q}$ indicated that 2 nd 
passage SP cells were more resistant to irradiation compared with 50th passage SP cells. As shown in Table III, 2nd passage cells displayed significantly larger values of $\mathrm{D}_{\mathrm{q}}$ than 50 th passage cells, indicating that the repair of sublethal damage capacity was diminished with increasing cell passage.

In addition, the doxorubicin and ionizing radiation-induced apoptosis in early and late passage cells were also analyzed. As shown in Fig. 4E, the apoptotic cell death rate was $7.94 \pm 0.82$ and $10.32 \pm 1.13 \%$ in early- and late-passage cells after 2 Gy irradiation, respectively. Following pre-incubation with $500 \mathrm{nM}$ doxorubicin, the apoptosis rate in 2nd and 50th passage cells was $22.76 \pm 2.81$ and $27.95 \pm 3.17 \%$, respectively (Fig. 4F). Similar results were observed in low and long-term passage NSCLC cells, where the apoptotic cell death rate was increased in the 50th passage cells (Fig. 4E and F).

\section{Discussion}

The CSC theory provides an attractive cellular mechanism counting for malignant progression and therapeutic resistance in lung cancer (5-10). The current anticancer therapies often destroy the bulk of a tumor mass but fail to eradicate CSCs (3). Thus, tumor recurrence or metastasis occurs by resident CSCs subsequent to a primary therapeutic response or initial induction of tumor remission (6-8). Therefore, a better understanding of CSCs biology will be useful to explore more effective methods to destroy CSCs.

In order to investigate the mechanism of CSCs resistance to anticancer therapy, the critical step is to isolate CSCs. The SP assay is based on the differential potential of cells to efflux Hoechst dye via an ABC transporter (15). The ability to exclude Hoechst dye as defined by SP fraction was initially described in mouse bone marrow cells, and later observed in haematopoietic malignancies and solid tumors such as lung cancer $(5,9,10,15)$. Increasing evidence suggests that a stem cell-like SP subpopulation exists in lung cancer cell lines, including A549, H23, H146, H460 and HTB-23, and in tumor biopsies $(5,9,10)$. Notably, the NSCLC cell line A549 used in the aforementioned studies exhibited a significantly different SP fraction, ranging from 2.59 to $24.44 \%(5,9,10)$. In addition, Salcido et al (9) observed that SCLC cell lines comprised $0.7-1.3 \%$ of SP cells, while the NSCLC cell lines A549 and H460 contained 2.59 and $4.00 \%$ of SP cells, respectively. The frequency of SP cells appears to be highly variable among different lung cancer cell lines of the same type. Accumulating evidence reveals the fact that repeated passaging of cell lines for multiple generations frequently leads to change of characteristics, including alterations in cell morphology, growth rates, protein expression and cell signaling, and acquisition of genetic aberrations (11-13). Thus, the distinct percentage of SP fraction may be associated with the different passaged cell line used in individual laboratories. In the present study, the results demonstrated that the SP fraction was slightly decreased after long-term passage within A549 and NSCLC cells.

Current approaches used to identify putative CSCs are based on the notion that CSCs should have conserved stem cell functions $(6,19,26)$, that is, CSCs are a functional definition. The methods used for the identification of isolated SP cells have been previously described $(6,8)$. Self-renewal was considered one of the basic features of stem cells (18). The present results suggest that 2nd passage A549 and NSCLC cells have increased capacity to form spheres compared with 50th passage cells, and this self-renewing ability was diminished after long-term passage. Consistent with the sphere formation results, the clonogenic assay revealed that the mean clone formation efficiency was decreased in long-term passage SP cells. The CSC theory postulates that CSCs exhibit characteristics similar to those of normal stem cells $(26,27)$, while RT-qPCR analysis revealed that long-term passage has no effects on the expression of ES genes. Collectively, those results suggested that long-term passage decreases the functional properties but not the ES expression of the stem cell-like SP fraction derived from A549 and NSCLC cells.

Tumor initiation and serial transplantation in immunocompromised mice was previously recommended as the golden standard for the identification of CSCs $(20,21)$. The present results suggested that the tumor-initiating frequency in 2 nd passage SP cells was higher than in 50th passage SP cells. The invasion capacity of CSCs has been frequently associated with the EMT $(28,29)$. E-cadherin and N-cadherin have been regarded as inducers of EMT (22). Loss of E-cadherin expression is emerging as one of the most common indicators of EMT onset, and its reduced expression in various cancers was previously associated with tumor progression and metastasis (22). N-cadherin was reported to be associated with an increased invasive potential in cancer (22). Snail has been described as a direct repressor of E-cadherin whose overexpression leads to a reduced expression of E-cadherin (29). A further molecule known to trigger EMT is Twist, and upregulation of Twist resulted in an increase of N-cadherin (23). RT-qPCR analysis indicated that N-cadherin, Snail and Twist genes were overexpressed, while E-cadherin expression was decreased, in 2nd passage cells compared with 50th passage cells. Those results indicated that aggressive malignancy features (such as tumor-initiating and migration abilities) of SP cells were inhibited by long-term passage.

According to the CSCs theory, current therapy options have limited capacities to destroy CSCs and tumor relapsed by resident CSCs $(24,25)$. The present study demonstrated that low passage cells were particularly resistant to ionizing radiation. Regarding doxorubicin sensitivity, low-passage cells only expressed significant resistance following a high dose of exposure. Apoptosis is the process of cell death in tumors following cancer treatment (such as chemotherapy and radiotherapy) (17,30,31). A previous study has revealed that the majority of therapy resistance results from failure of the cancer treatment to induce apoptosis pathways $(30,31)$. The present study revealed that 50th passage cells exhibit reduced doxorubicin and radiation-induced apoptotic cell death.

In summary, the present in vitro and in vivo studies demonstrated that CSC-like functional properties (such as sphere and clone formation efficiency, aggressive malignancy features and resistance to anticancer treatment) of long-term passage SP cells were diminished. However, the expression of ES genes was not affected by long-term passaging. This finding may partly explain the current confusing results, and suggests that low-passage cell lines and primary cancer cells should be used in the CSCs field. 


\section{References}

1. Jemal A, Bray F, Center MM, Ferlay J, Ward E and Forman D: Global cancer statistics. CA Cancer J Clin 61: 69-90, 2011.

2. Siegel R, Ma J, Zou Z and Jemal A: Cancer statistics, 2014. CA Cancer J Clin 64: 9-29, 2014.

3. Stewart BW and Kleihues P (eds): World Cancer Report 2003. IARC Press, Lyon, pp182-185, 2003.

4. García-Campelo R, Bernabé R, Cobo M Corral J, Coves J, Dómine M, Nadal E, Rodriguez-Abreu D, Viñolas $\mathrm{N}$ and Massuti B: SEOM clinical guidelines for the treatment of non-small cell lung cancer (NSCLC) 2015. Clin Transl Oncol 17: 1020-1029, 2015

5. Ho MM, Ng AV, Lam S and Hung JY: Side population in human lung cancer cell lines and tumors is enriched with stem-like cancer cells. Cancer Res 67: 4827-4833, 2007.

6. Gao YJ, Li B, Wu XY, Cui J and Han JK: Thyroid tumor-initiating cells: Increasing evidence and opportunities for anticancer therapy (review). Oncol Rep 31: 1035-1042, 2014.

7. Freitas DP, Teixeira CA, Santos-Silva F, Vasconcelos MH and Almeida GM: Therapy-induced enrichment of putative lung cancer stem-like cells. Int J Cancer 134: 1270-1278, 2014

8. Eramo A, Lotti F, Sette G, Pilozzi E, Biffoni M, Di Virgilio A, Conticello C, Ruco L, Peschle C and De Maria R: Identification and expansion of the tumorigenic lung cancer stem cell population. Cell Death Differ 15: 504-514, 2008.

9. Salcido CD, Larochelle A, Taylor BJ, Dunbar CE and Varticovski L: Molecular characterisation of side population cells with cancer stem cell-like characteristics in small-cell lung cancer. Br J Cancer 102: 1636-1644, 2010.

10. Sung JM, Cho HJ, Yi H, Lee CH, Kim HS, Kim DK, Abd El-Aty AM, Kim JS, Landowski CP, Hediger MA and Shin HC: Characterization of a stem cell population in lung cancer A549 cells. Biochem Biophys Res Commun 371: 163-167, 2008.

11. Sundareshan P and Hendrix MJ: Growth, morphologic, and invasive characteristics of early and late passages of a human endometrial carcinoma cell line (RL95-2). In Vitro Cell Dev Biol 28A: 544-552, 1992.

12. Wenger SL, Senft JR, Sargent LM, Bamezai R, Bairwa N and Grant SG: Comparison of established cell lines at different passages by karyotype and comparative genomic hybridization. Biosci Rep 24: 631-639, 2004.

13. Briske-Anderson MJ, Finley JW and Newman SM: The influence of culture time and passage number on the morphological and physiological development of Caco-2 cells. Proc Soc Exp Biol Med 214: 248-257, 1997.

14. Xie G, Zhan J, Tian Y, Liu Y, Chen Z, Ren C, Sun Q, Lian J, Chen L, Ruan J, et al: Mammosphere cells from high-passage MCF7 cell line show variable loss of tumorigenicity and radioresistance. Cancer Lett 316: 53-61, 2012.
15. Goodell MA, Brose K, Paradis G, Conner AS and Mulligan RC: Isolation and functional properties of murine hematopoietic stem cells that are replicating in vivo. J Exp Med 183: 1797-1806, 1996.

16. Schmittgen TD and Livak KJ: Analyzing real-time PCR data by the comparative C(T) method. Nat Protoc 3: 1101-1108, 2008.

17. Wu XY, Fan RT, Yan XH, Cui J, Xu JL, Gu H and Gao YJ: Endoplasmic reticulum stress protects human thyroid carcinoma cell lines against ionizing radiation-induced apoptosis. Mol Med Rep 11: 2341-2347, 2015.

18. Pastrana E, Silva-Vargas V and Doetsch F: Eyes wide open: A critical review of sphere-formation as an assay for stem cells. Cell Stem Cell 8: 486-498, 2011.

19. Zhang P, Zhang Y, Mao L, Zhang Z and Chen W: Side population in oral squamous cell carcinoma possesses tumor stem cell phenotypes. Cancer Lett 277: 227-234, 2009.

20. Ailles LE and Weissman IL: Cancer stem cells in solid tumors. Curr Opin Biotechnol 18: 460-466, 2007.

21. Clarke MF, Dick JE, Dirks PB, Eaves CJ, Jamieson $\mathrm{CH}$, Jones DL, Visvader J, Weissman IL and Wahl GM: Cancer stem cells-perspectives on current status and future directions: AACR Workshop on cancer stem cells. Cancer Res 66: 9339-9344, 2006.

22. Huber MA, Kraut $\mathrm{N}$ and Beug $\mathrm{H}$ : Molecular requirements for epithelial-mesenchymal transition during tumor progression. Curr Opin Cell Biol 17: 548-558, 2005.

23. Kang Y and Massagué J: Epithelial-mesenchymal transitions: Twist in development and metastasis. Cell 118: 277-279, 2004.

24. Milas L and Hittelman WN: Cancer stem cells and tumor response to therapy: Current problems and future prospects. Semin Radiat Oncol 19: 96-105, 2009.

25. Pajonk F, Vlashi E and McBride WH: Radiation resistance of cancer stem cells: The 4 R's of radiobiology revisited. Stem Cells 28: 639-648, 2010

26. Ratajczak MZ: Cancer stem cells-normal stem cells 'Jedi' that went over to the 'dark side'. Folia Histochem Cytobiol 43: 175-181, 2005.

27. Dalerba P, Cho RW and Clarke MF: Cancer stem cells: Models and concepts. Annu Rev Med 58: 267-284, 2007.

28. Nurwidya F, Takahashi F, Murakami A and Takahashi K: Epithelial mesenchymal transition in drug resistance and metastasis of lung cancer. Cancer Res Treat 44: 151-156, 2012.

29. Cano A, Pérez-Moreno MA, Rodrigo I, Locascio A, Blanco MJ, del Barrio MG, Portillo F and Nieto MA: The transcription factor snail controls epithelial-mesenchymal transitions by repressing E-cadherin expression. Nat Cell Biol 2: 76-83, 2000.

30. Verheij M and Bartelink H: Radiation-induced apoptosis. Cell Tissue Res 301: 133-142, 2000.

31. Kaufmann SH and Earnshaw WC: Induction of apoptosis by cancer chemotherapy. Exp Cell Res 256: 42-49, 2000. 